\title{
Chaotic Duck Traveler Optimization (cDTO) Algorithm for Feature Selection in Breast Cancer Dataset Problem
}

\section{Krishnaveni Arumugam ${ }^{1}$, Shankar Ramasamy ${ }^{\text {b* }}$, Duraisamy Subramanic ${ }^{*}$}

aph.D Research Scholar, ${ }^{\text {b* \& }}{ }^{\text {c\#} A s s i s t a n t ~ P r o f e s s o r s ~}$

a, b*\&c\# Department of Computer Science, Chikkanna Govt. Arts College, Tiruppur.

Article History: Received:11 January 2021; Accepted: 27 February 2021; Published online: 5 April 2021

\begin{abstract}
Objective: 1 of every 3 individuals will be determined to have malignancy in the course of their life. Currently, there are more than 3.8 million ladies who have been determined to have breast malignancy in the United States. 2021 is practically around the bend, yet there's still an ideal opportunity to help ladies confronting breast malignancy in 2020. In this paper, chaotic based duck travel optimization (cDTO) meta-heuristic algorithm is introduced to classifying the input images from Mammogram Image Analysis Society (MIAS) database. Methods: Linear Discriminant Analysis is used to extract the mammogram image features. (cDTO-LDA) is an intrinsic algorithm to remove irrelevant features and select the optimal features by using wavelet families Haar (harr), db4 (daubechies), bior4.4 (Biorthogonal), Symlets (SYM8), "Discrete" FIR approximation of Meyer wavelet (dmey) features. Results: These selected features are evaluated by the quality measures such as accuracy, sensitivity, specificity, error rate that are clearly shows the high exactness of cDTO classifier is 98.5\%. CSA-LDA classifier has the minimum exactness. Conclusion: Algorithm efficiency is proved by the promising results achieved by the proposed algorithm for selecting the best feature of breast cancer classification.
\end{abstract}

Keywords: Bio Inspired Algorithm, Chaotic Duck Traveler Optimization, Breast Tumor, LDA, Accuracy

\section{I) Introduction}

Clinical picture preparing assumes a vital part in disease conclusion and anticipation. Everybody conveys BRCA 1 and BRCA 2 genes in their cells. Typically, these qualities work to help fix harm to DNA, which happens each day. With an acquired BRCA gene transformation, damaged DNA may not be fixed appropriately. This locates individuals with a mutation at an expanded danger for building up specific kinds of malignancy, including breast disease. Sentinel lymph node (SLN) is the principal phlegm to get leakage straightforwardly from a tumor [1]. In 2020 breast disease survey shows those roughly 2261419 new cases (11.7\%) in determination stage and Breast malignant growth is as yet one of the main sources of malignant growth passing in ladies 684996 new cases (6.9\%) in death stage [2]. Malignant growth that begins in the breast is known as breast tumor. After cellular breakdown in the lungs (exclusive of skin diseases), Breast cancer (C50) is the most widely recognized disease analyzed among US ladies and is the second leading reason for malignant growth rate of death. Prognosis for the breast cancer classification includes early discovery and treatment [3]. The wide-ranging levels of risk for breast cancer have important implications for safe than death. Self awareness is needed to live a happy and protected life without breast tumor (BT) in a lifelong. [4]. Many authors find that Meta heuristic algorithms based on trial-and-error method is used to early detection [5].

A great situate to initiate away learning scientific image is within the field of medical image processing, since it involves algorithms that smooth the progress of exchange information into pictures [6]. In the course of recent many years a remarkable interest and progress in nonlinear frameworks, chaos theory, and fractals have been seen, which are reflected in the classification of diseases. Wolfram Math world characterize chaos is a deterministic advancement of nonlinear frameworks. Exponential sensitivity (Chaos) to little perturbations shows up wherever in nature at powerfully [7]. Choosing the most discriminative highlights is a difficult issue in numerous applications. Bio-inspired computing calculations in optimization have been generally applied to tackle numerous optimization issues including the component choice issue. Assortment of data is called as information. Without information, everybody in this world can't speak with each other. Information might be excess,loud, unimportant, and complex to comprehend by somebody. So diminish the dimensionality of information is required for include choice in arrangement [8]. 


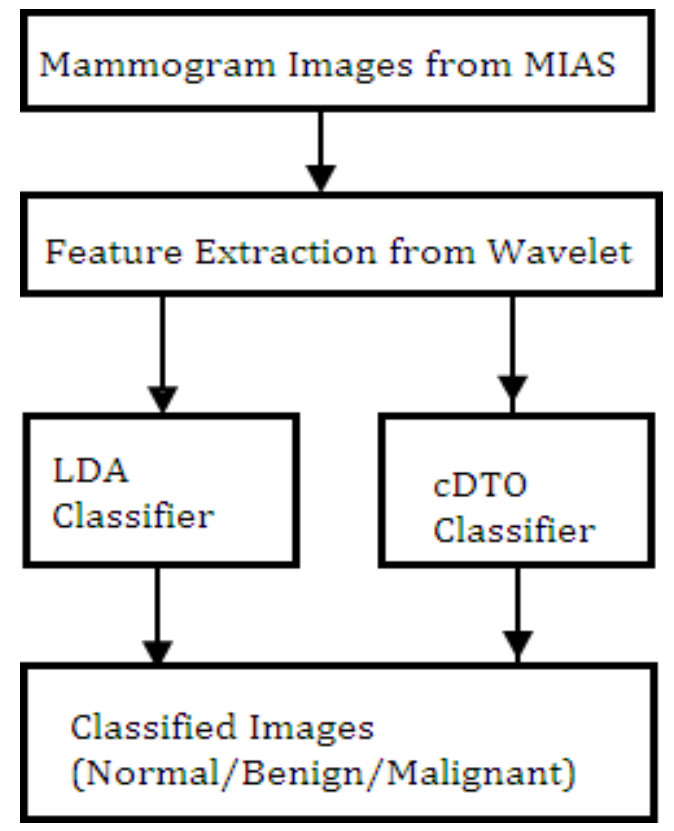

Fig 1 Classification methodology of proposed cDTO for mammogram images

\section{II) Background Study}

Ogivri Trusted Source (trastuzumab-dkst), is affirmed by the FDA for bosom malignant growth treatment. In contrast to generics, bio similars are duplicates of biologic medications and cost not exactly marked medications. In 2018 A clinical preliminary proposes that chemotherapy after medical procedure doesn't profit $70 \%$ of ladies with beginning phase bosom malignant growth. In 2019 Enhertu Trusted Source is affirmed by the FDA, and this medication ends up being viable in treating HER2-positive bosom disease that is metastasized or can't be taken out with a medical procedure. In $\mathbf{2 0 2 0}$ the medication Trodelvy is endorsed by the FDA for treating metastatic triple-pessimistic bosom malignancy for individuals who haven't reacted to in any event two different medicines [9].

The authors Chiranjib Sur et al determine the chatic based EVO algorithm in 2013. In 2014, Gandomi et al explains chaotic based ISA algorithm. In 2016 javidi et al proposed chaotic PSO for feature extraction. In the same year zawbaa et al introduced chaotic ALO and Wang et al presents chaotic CS for classification purpose. Similarly in 2017 Ahmed et al expounds chaotic CSO. In 2018 Sayed et al proposed chaotic WOA and introduced chaotic CSA in 2019. The author's ewees et al introduced chaotic based MVO for classification. In ref [11] the creators used chaos theory based on molecular cloud and find out the optimal species in the cloud.

This paper presents two distinctive chaotic forms of fundamental chicken swarm optimization calculation utilizing tent and coordination's map; logistic map with chicken multitude presents the best outcomes for include determination against four benchmark models with five quality measures. The proposed chaotic chicken multitude calculation (CCSO) based element determination calculation is contrasted and four feature selection calculations on five benchmark informational data sets. An examination among a few sorts of well known classifiers is never really out the sensitivity of every classifier relating to the selected highlights and the measurement decrease. During iterations, the best wellness value shows wonderful improvement of the grouping exactness [12].

In ref [13] assessed method utilizing diverse chaotic guide on various component choice datasets. To guarantee generality, they utilized ten natural datasets, yet they additionally utilized different kinds of information from different sources. The outcomes are contrasted and the molecule swarm enhancer and with hereditary calculation variations for highlight choice utilizing a bunch of value measurements [13]. 
In this paper, a novel Meta heuristic enhancer, to be specific chaotic crow search calculation (CCSA), is proposed to beat nearby optima issues. The proposed CCSA is applied to enhance include determination issue for 20 benchmark datasets. Ten chaotic guides are utilized during the enhancement cycle of CSA. The presentation of CCSA is contrasted and other notable and late enhancement calculations. Test results uncover the capacity of CCSA to locate an ideal component subset which amplifies the grouping execution and limits the quantity of chose highlights. Besides, the outcomes show that CCSA is better looked at than CSA and different calculations. Moreover, the investigations show that sine chaotic map is the fitting guide to essentially support the exhibition of CSA [14].

This paper gives a novel chaotic MVO calculation (CMVO) to evade slow convergence, where chaotic guides are utilized to improve the exhibition of MVO calculation. The CMVO calculation is applied to take care of the component determination issue, in which five benchmark datasets are utilized to assess the presentation of CMVO calculation. The consequences of CMVO are contrasted and standard MVO and two other multitude calculations. The exploratory outcomes show that calculated tumultuous guide is the best riotous guide that builds the presentation of MVO, and furthermore the MVO is superior to other multitude calculations [15].

\section{III) Materials and Methods}

\section{A) Dataset (MIAS)}

Breast Cancer (C50) is classified as normal or benign or malignant by using the proposed c-DTO with LDA Classifier efficiently. Mammogram Image Analysis Society (MIAS) dataset contains 322 pictures with the blend of left and right breast. The pictures are ordered into three primary gatherings as thick glandular, greasy and greasy glandular bosoms dependent on the qualities of breast tissue. The pictures are further classified into three significant classifications dependent on the presence of calcification: malignant, benign and normal [10]. Chest picture contains radio-misty antiques, for example, wedges and marks. Now and again it contains a few patients' individual data. The pictures are preprocessed to eliminate the wedges, marks and noise present in it during the acquisition. MATLAB 2015a is used for all the specified experiments in this paper.

Here, the proposed cDTO with different classifiers for breast cancer diagnosis is described in detail. Initially, breast input data is collected and then the feature selection techniques (LDA) is utilized to select the most significant features. The selected features are given as input to cDTO for breast cancer classification. Feature selection inherits the significant features from parent class to child class of the obtainable information. Meta heuristic with binary or chaotic performs role play to minimize the false positive rate, decrease data dimensionality, decline cost expensive and maximize the accuracy for best optimal feature selection and extraction.

\section{B) Feature Extraction}

In image processing feature extraction plays an important role in dimensionality reduction without loss of any important data. Selecting the essential feature without knowing the irrelevant feature is similar to the data abstraction. The feature extraction and optimal feature selection from an input image plays a critical role in the performance of LDA Classifier. Higher accuracy is obtained by LDA classifier with chaotic based DTO algorithm. LDA select the optimal features by using wavelet families Haar (harr), db4 (daubechies), bior4.4 (Biorthogonal), Symlets (SYM8), "Discrete" FIR approximation of Meyer wavelet (dmey) features. This wavelet features offers the statistical features like accuracy, error rate, Mathews correlation coefficient, precision, sensitivity, specificity, mean, contrast, smoothness, standard deviation and skewness.

In the earliest prediction of breast cancer, tumor region segmentation, benign and malignant that is classifying cancerous or non cancerous process are successfully done by using CAD (Computer Aided Systems). In object oriented programming the grouping of objects is known as class. Similarly in image, 'partitioning' homogeneous parts of an object is called as image segmentation. Segmentation plays an important role in the field of medical image analysis, computer vision, machine learning, network security and cryptography etc. For medical image processing especially tumor region segmentation, Seeded Region Growing (SRG) is the preferred method, Thresholding, Histogram, and Clustering also applied for preprocessing the input image for enhancement. 
a) Mean

Calculate the average value from total pixels of an image.

$$
\text { Mean }=\sum_{\mathrm{k}=\mathrm{O}_{\mathrm{K}}}^{\mathrm{L}-1} \mathrm{X}_{\mathrm{k}} * \mathrm{P}\left(\mathrm{X}_{\mathrm{k}}\right)
$$

b) Standard Deviation

The Standard Deviation is a proportion of how spreads out numbers are.

c) Contrast

$$
\text { Standard Deviation }=\underset{\mathrm{L}-1}{\sum\left(\mathrm{X}_{\mathrm{k}}-\mathrm{a}\right)^{2} * \mathrm{P}\left(\mathrm{X}_{\mathrm{k}}\right)}\left(\begin{array}{c}
\mathrm{k}= \\
\mathrm{O}
\end{array}\right.
$$

Brightness and darkness of the picture is adjusted by the user.

d) Smoothness

$$
\text { Contrast }=\underset{\substack{\mathrm{L}-1 \\ \mathrm{k}=\mathrm{O}}}{\sum \sqrt{ }\left(\mathrm{X}_{\mathrm{k}}-\mathrm{a}\right)^{2} * \mathrm{P}\left(\mathrm{X}_{\mathrm{k}}\right)}
$$

It Measures the relative force varieties in a locale.

e) Skewness

$$
\text { Smoothness }=1-\frac{1}{(1+a)^{2}}
$$

Skewness demonstrates positive numbers as results.

$$
\text { Skewness }=\underset{\mathrm{k}=\mathrm{O}}{\sum_{\mathrm{L}}-1}\left(\mathrm{X}_{\mathrm{k}}-\mathrm{a}\right)^{3} * \mathrm{P}\left(\mathrm{X}_{\mathrm{k}}\right)
$$

f) Kurtosis

Highest value of mean is considered as kurtosis value.

$$
\text { Kurtosis } \underset{k=O}{\operatorname{L}-1}\left(\mathrm{X}_{\mathrm{k}}-\mathrm{a}\right)^{4} * \mathrm{P}\left(\mathrm{X}_{\mathrm{k}}\right)
$$

g) Accuracy

Correctly classified occurrences for all instances is measured by accuracy as below

\section{h) Precision}

$$
\text { Accuracy }=\frac{\mathrm{TP}+\mathrm{TN}}{\mathrm{TP}+\mathrm{FP}+\mathrm{TN}+\mathrm{FN}}
$$

Correctly classified occurrences for those instances is measured as optimistic value calculated by

$$
\text { Precision }=\frac{\text { TP TP }}{+ \text { FP }}
$$




\section{C) Classification using BIRADS Score}

The radiologist appoints a solitary digit Breast Imaging Reporting and Data System score (going from 0 to 5) when the report of women's mammogram is made.

Table 1 BIRADS Classification of malignancy likelihood score and its recommendation

\begin{tabular}{|l|l|l|l|}
\hline $\begin{array}{l}\text { BIRADS } \\
\text { CATEGORY }\end{array}$ & DESCRIPTION & $\begin{array}{l}\text { MALIGNANCY } \\
\text { LIKELIHOOD } \\
\text { SCORE }\end{array}$ & RECOMMENDATION \\
\hline BIRADS 0 & Need Sufficient Detail & Two-Ten\% & Revised \\
\hline BIRADS One & Standard & Within zero. one\% & Periodic Revising \\
\hline BIRADS 2 & Compassionate & Within zero. one\% & Periodic care \\
\hline BIRADS 3 & Doubtless & Within one. eight $\%$ & Stipulated time care is needed \\
\hline BIRADS 4 & Highly suspicious & $10-55 \%$ & Biospy \\
\hline BIRADS 5 & Malignant & $60-100 \%$ & Biospy \\
\hline BIRADS 6 & Known Cancer & $100 \%$ & Treat Malignancy \\
\hline
\end{tabular}

\section{D) Classification using LDA}

LDA is similar to PCA. In this research paper, utilizing Linear Discriminant Analysis (LDA), an endeavor is made towards effectively anticipating the class of breast cancer (benign or malignant) and to assist specialists with diagnosing illness at a beginning phase to diminish the danger of fatal disease. Notwithstanding its straightforwardness, LDA frequently delivers hearty, nice, and interpretable order results.

in addition finds the arcs that maximize the separation between classes.

$$
\left.\left.\boldsymbol{\beta}^{\mathbf{T}}\left(\mathbf{X}-\frac{(\mu 1+\mu 1}{2}\right)\right)>\log \frac{\mathrm{P}(\mathrm{C} 1)}{\mathrm{P}(\mathrm{C} 2}\right)
$$

$\beta$ is a coefficient vector and $X$ is a data vector. $P$ represents class probability.
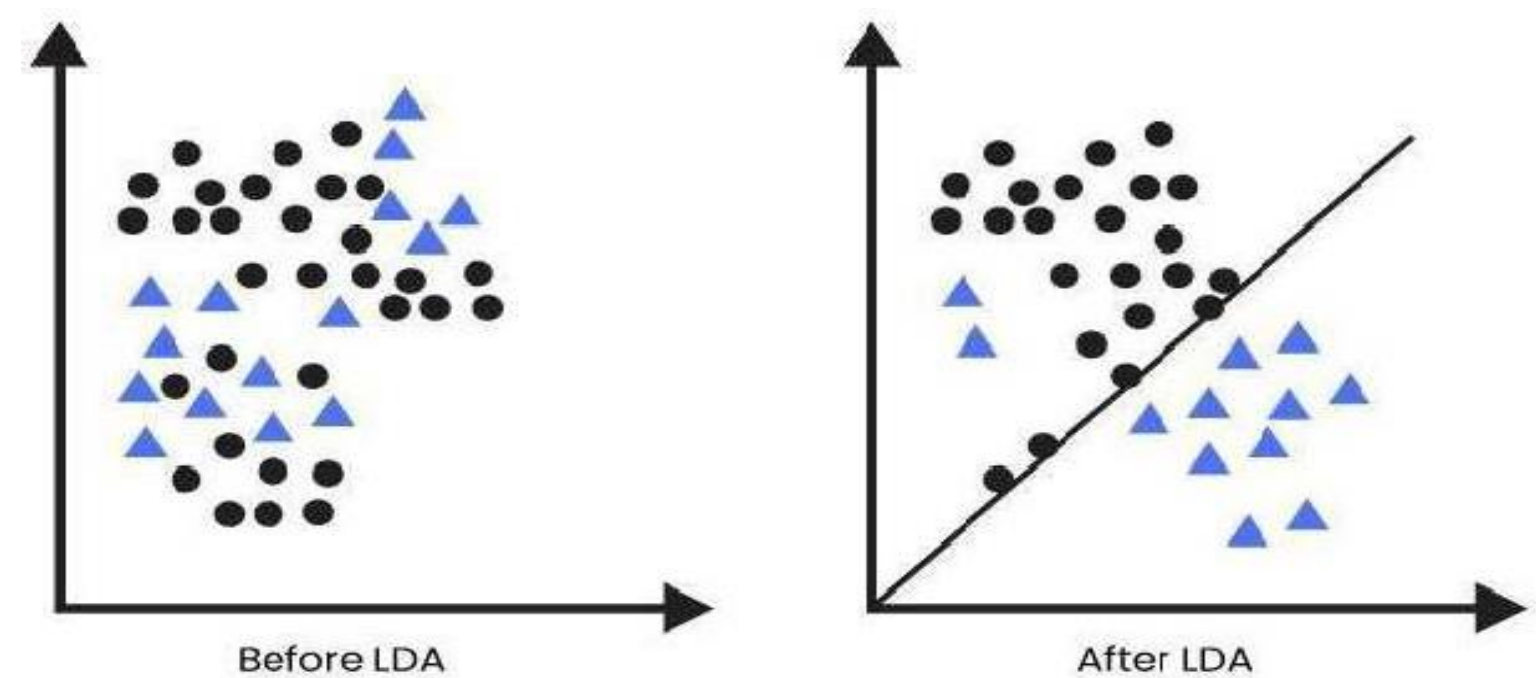

Fig 2 Classification using LDA

\section{E) Classification using cDTO}

Chaotic strategies have significant properties, for example, ergodicity, stochastically natural, and indicating irregular conduct just as delicate reliance on the underlying conditions. These properties have been meant different conditions which are 
called "chaotic guides" to be relevant for utilizing in computational applications, for example, optimization issue. In this way, utilizing these guides to refresh arbitrary factors in enhancement strategies is called chaotic optimization algorithm (COA). This change causes enhancement strategies to acquire the strength of chaos, for example, the ergodic and non repetition; along these lines, it can escape from neighborhood optima and accomplish rapid inquiries than random search.

$$
\mathrm{C}(\mathrm{t}+1)=4 * \mathrm{C}(\mathrm{t}) *(1-\mathrm{C}(\mathrm{t}) \mathrm{C}(\mathrm{t}) \in(0,1)
$$

If initial weight value $\mathrm{C}=0$ duck flock comprises a swimming in water fowl. If $\mathrm{C}=1$ then all the ducks flying in air. Chaotic weight value of DTO is called Chaotic Duck Traveler Optimization (cDTO) algorithm

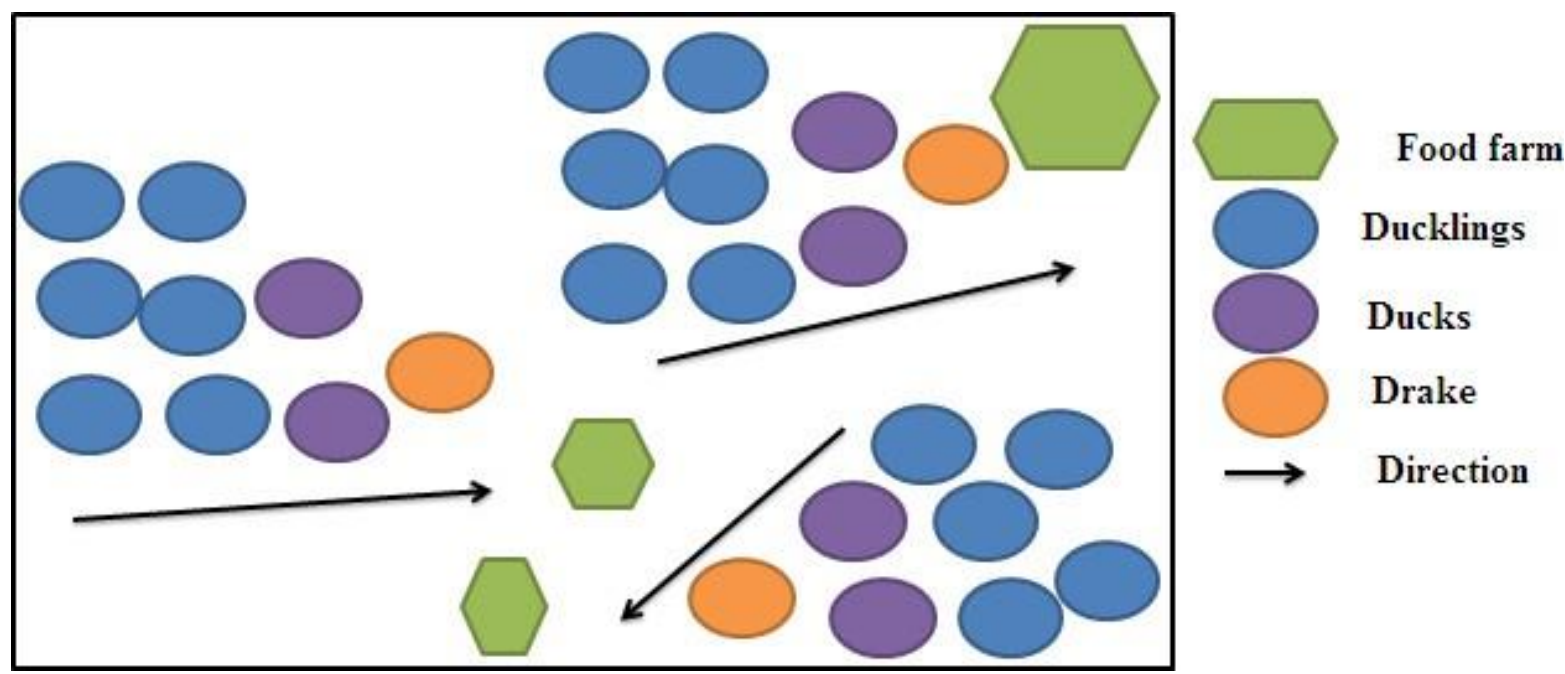

Fig 3 finding the food farm by chaotic duck flock

Figure 3 Drake in each duck flock guiding the direction to its two ducks and ducklings to reach the food farm.

Ducks are exceptionally social creatures and this implies they need different ducks to live with. Ducks straight is a key motivation which goes about as take it to the following level. That implies things are efficient. All the little subtleties are represented and are in their appropriate situations before proceeding onward to something different.

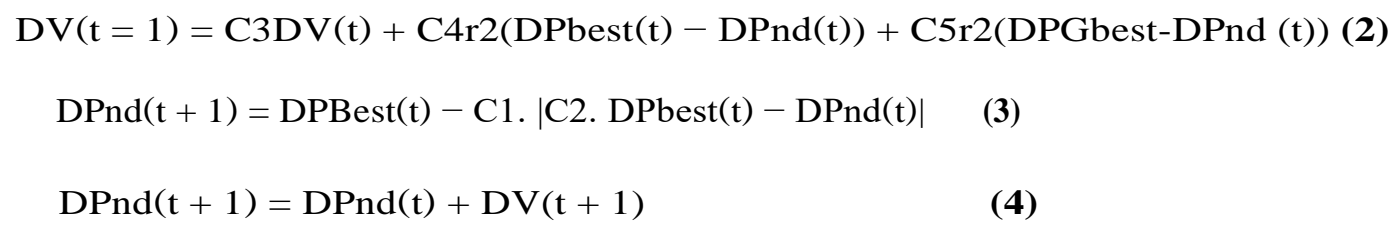

$\Delta \mathrm{D}^{\mathrm{i}} \mathrm{t}+1=\mathrm{h} \Delta \mathrm{Dti}+\mathrm{C} 1 \mathbf{r} 1\left(\right.$ DPebest $\left._{\mathrm{ti}}-\mathrm{Dti}\right)+\mathrm{C} 2 \mathrm{r} 2\left(\right.$ DPGbest $\left._{\mathrm{ti}}-\mathrm{Dti}\right)$

(5)

$\mathrm{D}^{\mathrm{i}} \mathrm{t}+1=\mathrm{D}_{\mathrm{t}}^{\mathrm{i}}+\Delta \mathrm{D}^{\mathrm{i}} \mathrm{t}+1$

(6)

\section{cDTO Algorithm}

Initialize the duck population $\mathrm{Di} i=1,2, \ldots \mathrm{n}$, maximum iteration Nxit, $\mathrm{t}=0$, number of search ducks $\mathrm{S}$, maximum number of search agents $\mathrm{S}_{\mathrm{Nax}}$. 
Initialize $\mathrm{D}_{\mathrm{i}}$ with features of breast data

while the stop condition is not pleased

for each duck

Call bDTO/SAF/LDA to find the classification accuracy

if classification accuracy $<$ DP-pbest

Travel with the present assessment to DP-pbestmatrix

endif

if classification accuracy $<$ DP-Gbest

Allocate present value as DP-Gbest

endif

end for

For each duck

Revise the groceries and predator

Revise fn, c, C1, C2, C3, C4, C5,

$\mathrm{r} 1, \mathrm{r} 2$

Compute objective function $\mathrm{f}_{\mathrm{n}}$ for each duck $\mathrm{DP}_{\mathrm{i}}$

Revise neighboring radius $\mathrm{R}$

if a duck has at least one adjacent duck

Revise velocity vector using (2)

Revise position vector using (3)

else

Revise position vector using (4)

end if

According to the changeable restrictions ensure and approved the original locations

end while

For each duck in a duck flock

Initialize ducks with DP-pbestmatrix

Allocate DP-Gbest

end

While Nxit is not attained

For each duck

Call bDTO/SAF/LDA to find the classification accuracy

if classification accuracy<DP-pbest in the past

Allocate present assessment as the original DP-pbest

end if

end for

Pick the duck with the best fitness value of duck flock in DT-Gbest

For each duck

Speed calculated by using (5)

Location changed by utilizing (6)

end For

end While

best-fitness=PSO-gbest

end while 


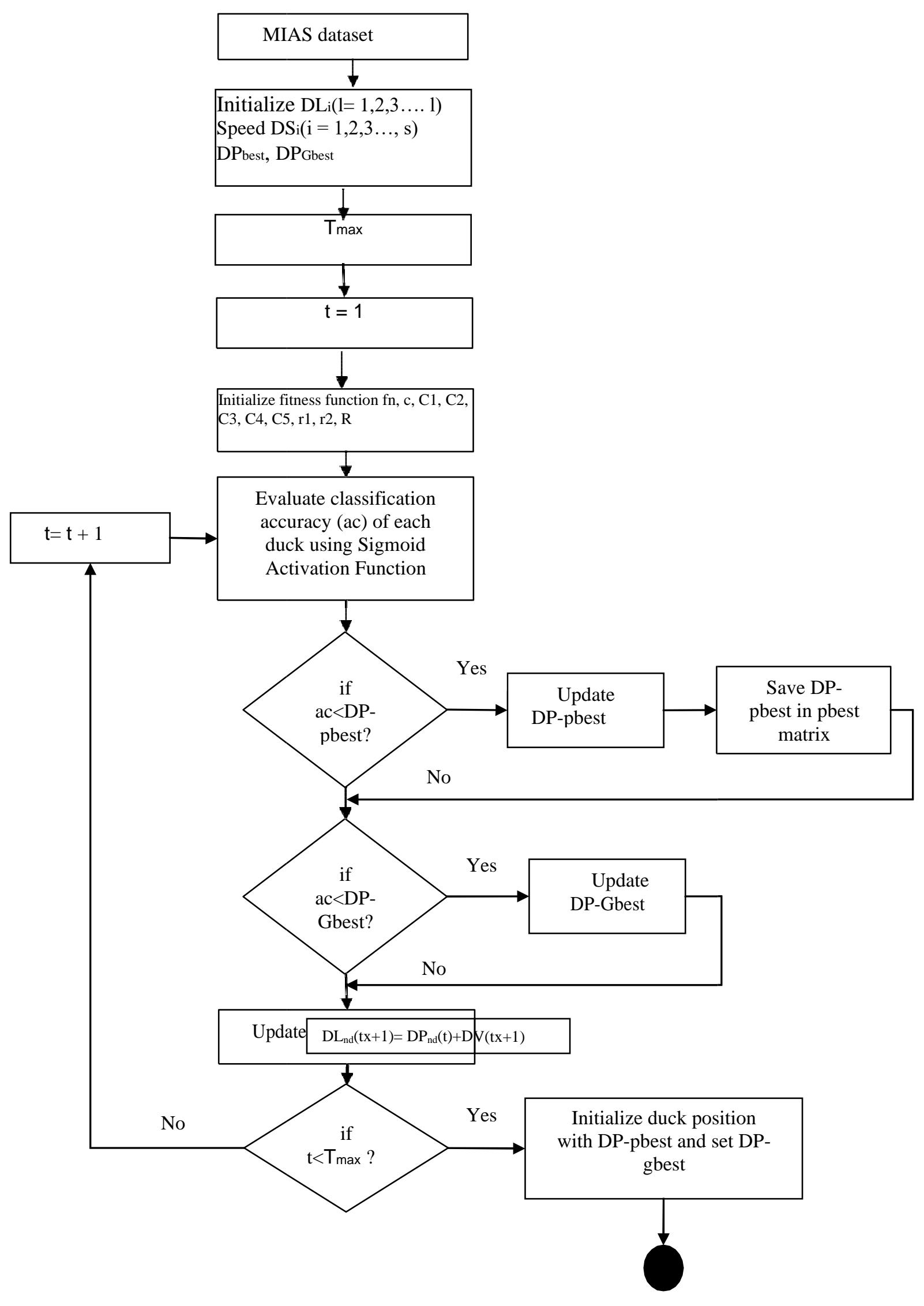




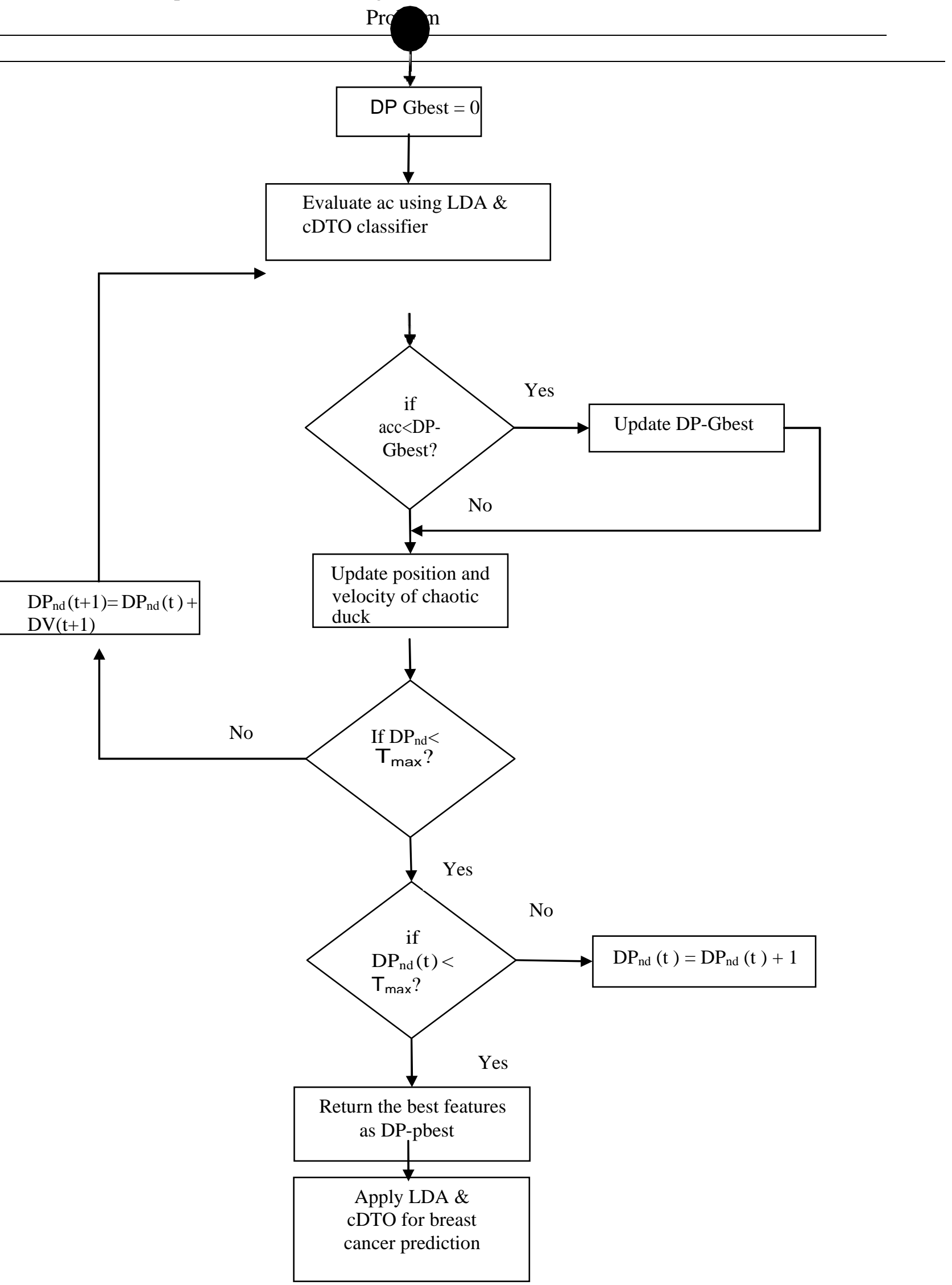

Fig 4 Workflow of chaotic duck with different classifier for breast cancer

The dynamic and static swarming activities represent discovery and manipulation quite closely to two main phases of meta-heuristic optimization. The static swarm is complimentary in the exploration phase while the dynamic swarm is complimentary in the exploitation phase. The exploration and exploitation are replicated either statistically or dynamically probing for optimal features or remove the irrelevant features in the gene expression data. 

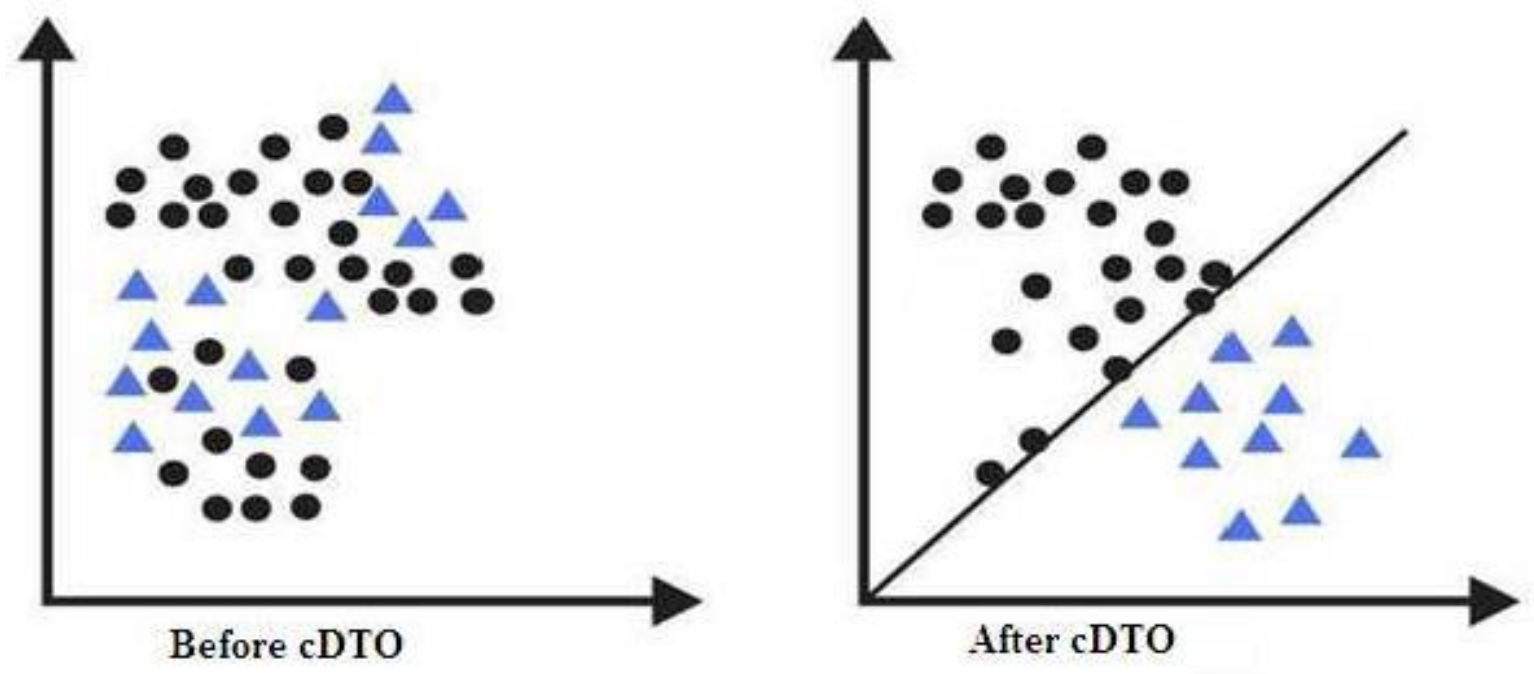

Fig 5 Classification using cDTO

IV) Experimental Results

Table 2 Two Classifiers Confusion matrix- LDA \& cDTO

\begin{tabular}{|l|l|c|c|c|c|c|}
\hline \multicolumn{2}{|c|}{ Classifiers } & \multicolumn{5}{c|}{ Wavelet Family Features } \\
\hline \multirow{2}{*}{$\begin{array}{l}\text { Linear Discriminant Analysis \& } \\
\text { Chaotic Duck Traveler Optimization }\end{array}$} & Daubechies (Db4) & Haar & Bior Splines (4.4) & Symlet 8 & Dmeyer \\
\hline \multirow{4}{*}{ LDA } & True Positive & 26 & 32 & 34 & 32 & 30 \\
\cline { 2 - 7 } & False Positive & 10 & 6 & 12 & 4 & 16 \\
\cline { 2 - 7 } & True Negative & 30 & 34 & 28 & 36 & 24 \\
\cline { 2 - 7 } & False Negative & 14 & 8 & 6 & 8 & 10 \\
\hline \multirow{4}{*}{ cDTO } & True Positive & 28 & 40 & 36 & 36 & 34 \\
\cline { 2 - 7 } & False Positive & 10 & 2 & 14 & 6 & 8 \\
\cline { 2 - 7 } & True Negative & 30 & 38 & 26 & 34 & 32 \\
\cline { 2 - 7 } & False Negative & 12 & 0 & 4 & 4 & 6 \\
\hline
\end{tabular}

Table 3 Two Classifiers Quality metrics for evaluation of performances- LDA \& cDO

\begin{tabular}{|l|l|c|c|c|c|c|}
\hline \multicolumn{2}{|c|}{ Classifiers } & \multicolumn{5}{|c|}{ Wavelet Family Features } \\
\hline $\begin{array}{l}\text { Linear Discriminant Analysis \& } \\
\text { Chaotic Duck Traveler Optimization }\end{array}$ & Daubechies (Db4) & Haar & Bior Splines (4.4) & Symlet 8 & Dmeyer \\
\hline \multirow{4}{*}{ LDA } & Accuracy & 70 & 82.5 & 77.5 & 85 & 67.5 \\
\cline { 2 - 7 } & Error Rate & 30 & 17.5 & 22.5 & 15 & 32.5 \\
\cline { 2 - 7 } & $\begin{array}{l}\text { Mathews Correlation } \\
\text { Coefficient }\end{array}$ & 40.20 & 65.08 & 55.63 & 70.35 & 35.40 \\
\cline { 2 - 7 } & Precision & 72.22 & 84.21 & 73.91 & 88.89 & 65.22 \\
\cline { 2 - 7 } & Sensitivity & 65 & 80 & 85 & 80 & 75 \\
\cline { 2 - 7 } & Specificity & 75 & 85 & 70 & 90 & 60 \\
\hline \multirow{5}{*}{ cDTO } & Accuracy & 72.5 & 98.2 & 77.5 & 87.5 & 82.5 \\
\cline { 2 - 7 } & Error Rate & 27.5 & 2.5 & 22.5 & 12.5 & 17.5 \\
\cline { 2 - 7 } & $\begin{array}{l}\text { Mathews Correlation } \\
\text { Coefficient }\end{array}$ & 45.6 & 95.12 & 56.80 & 75.09 & 65.08 \\
\cline { 2 - 7 } & Precision & 73.68 & 95.24 & 72 & 85.71 & 80.95 \\
\cline { 2 - 7 } & Sensitivity & 70 & 100 & 90 & 90 & 85 \\
\cline { 2 - 7 } & Specificity & 75 & 95 & 65 & 85 & 80 \\
\hline
\end{tabular}


As per the table results, the proposed LDA classifier is used for classifying the breast tumor and identified with high exactness $98.2 \%$.

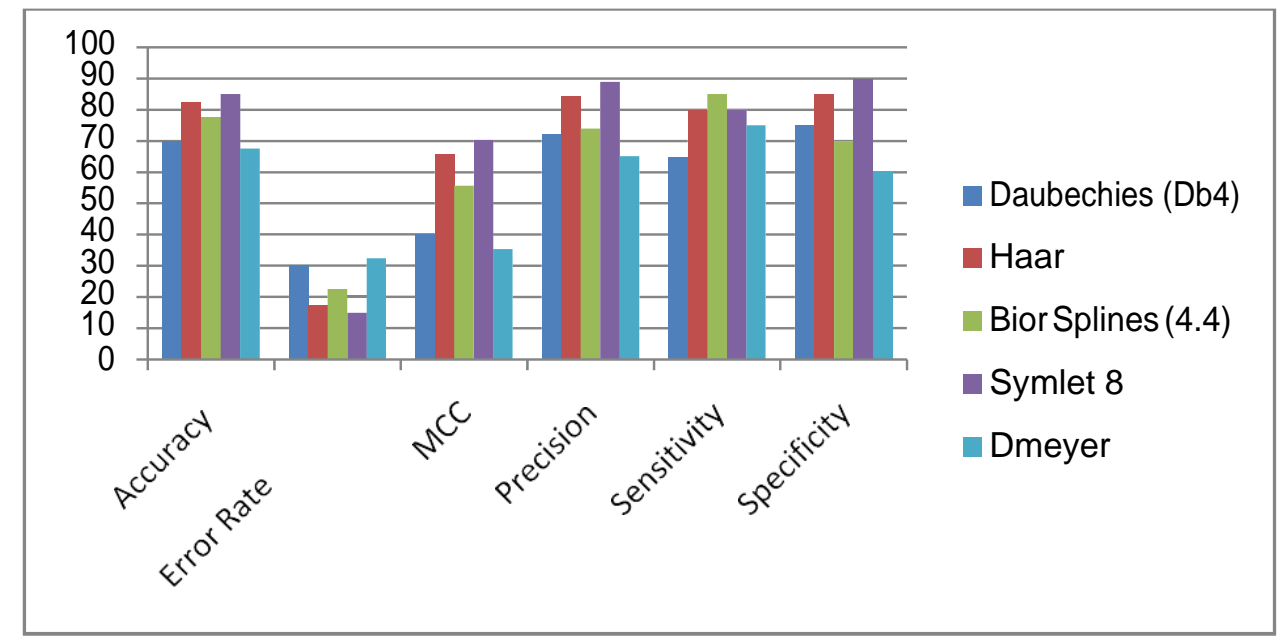

Fig 6 LDA Classifier Performance Evaluation

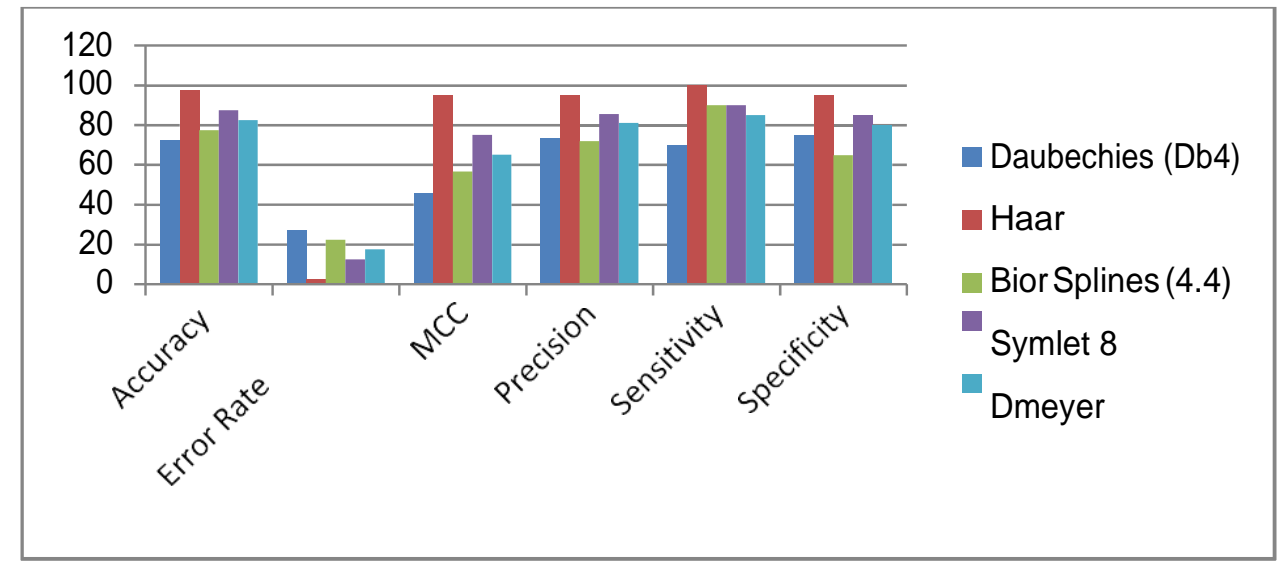

Fig 7 cDTO Classifier Performance Evaluation

V) Discussion

Table 4 Comparison analysis of proposed work with related existing work

\begin{tabular}{|c|c|c|c|c|}
\hline Authors & Reference & Year & Techniques & $\begin{array}{c}\text { Classification } \\
\text { Accuracy }(\%)\end{array}$ \\
\hline Srivastava et al & {$[18]$} & 2014 & $\begin{array}{c}\text { Hybrid features with } \\
\text { KNN }\end{array}$ & 87 \\
\hline Pratiwi et al & {$[19]$} & 2015 & $\begin{array}{c}\text { Texture features with } \\
\text { RBFNN }\end{array}$ & $\begin{array}{c}\text { Texture features with } \\
\text { ANN }\end{array}$ \\
\hline Saini et al & {$[20]$} & 2015 & Wavelet features with \\
GFS & 89.98 \\
\hline Pawar et al & {$[21]$} & 2016 & $\begin{array}{c}\text { Texture features with } \\
\text { Sparse representation }\end{array}$ & 93.75 \\
\hline Vaidehi et al & {$[22]$} & 2017 & $\begin{array}{c}\text { Texture features with } \\
\text { BPNN }\end{array}$ & 96.3 \\
\hline Gautam et al & {$[23]$} & 2018 & $\begin{array}{c}\text { Statistical features with } \\
\text { CSA }\end{array}$ & 97.5 \\
\hline Sannasi et al & {$[24]$} & 2019 & $\begin{array}{c}\text { Statistical features with } \\
\text { CNN }\end{array}$ & $92 \%$ \\
\hline $\begin{array}{c}\text { Shelembijaphet et al } \\
\text { Our proposed research } \\
\text { work }\end{array}$ & {$[25]$} & 2020 & $\begin{array}{c}\text { Statistical features with } \\
\text { cDTOA }\end{array}$ & 98.2 \\
\hline
\end{tabular}


The proposed cDTO algorithm for mammographic breast tumor classification accomplishes better outcomes demonstrated with accuracy of 98.2\% when compared with what was accounted for by Srivastava et al [18], Pratiwi et al [19], Saini et al [20], Pawar et al [21], Vaidehi et al [22], Gautam et al [23], Sannasi et al [24], Shelembijaphet et al [25].

\section{VI) Conclusion}

To distinguish breast disease standard or dangerous dependent on CAD mammogram pictures is done in this research paper. Because of improved outcome (accuracy) acquired from the framework, the proposed study presents Computer aided design framework to help radiologist in identifying the condition of bosom from mammograms naturally. As main contribution to the Linear Discriminant Analysis (LDA) and Chaotic Duck Traveler Optimization (cDTO) classifiers individually perform very well. The perfection idea of haar wavelet alongside cDTO classifier gives an improved accuracy of $13.2 \%$ when contrasted and LDA classifier. By utilizing cDTO calculation, the correlation of three plans of mammogram characterization (Normal/Benign/Malignant) was finished.

\section{References}

1. N.U.Dogan, S.D.G.Favero, C.Kohler, P.Dursun, "The basics of sentinel lymph node biopsy: Anatomical and pathophysiological considerations and clinical aspects", Journal of Oncology, Hindawi, 10 pages (2019) https://doi.org/10.1155/2019/3415630

2. C.E.DeSantis, J.Ma, M.M.Gaudet, L.A.Newman, K.D.Miller, A.G.Sauer, A.Jernal, R.L.Siegel, "Breast Cancer Statistics" (2019)

3. https://www.uicc.org/what-we-do/thematic-areas-work/breast-cancer

4. E.L.Henriksen, J.F.Carlsen, I.M.M.Vejborg, M.B.Nielsen, C.A.Lauridsen, "The efficacy of using computer-aided detection (CAD) for detection of breast cancer in mammography screening: a systematic review" Acta radiologica volume:60, issue(1) pages:13-18 (2019)

5. Krishnaveni A, Shankar R, Duraisamy S, "A Survey on Nature Inspired Computing (NIC): Algorithms and Challenges", Global journal of computer science and technology: D Neural \& Artificial Intelligence volume 19 issue 3 version 1.0 Year (2019).

6. A.Krishnaveni, R. Shankar and S. Duraisamy, "A Review on various Image Thresholding Methods for Mammogram Image Segmentation” Compliance Engineering Journal ISSN NO: 0898-3577 Volume 11, Issue 2, (2020).

7. D.Toker, F.T.Sommer, M.D.Esposito, "A Simple Method for chaos in nature", Communications Biology volume:3 (2020)

8. A.A.Nahid, M.A.Mehrabi, Y.Kong, "Histopathological Breast Cancer Image Classification by deep neural network techniques guided by local clustering", Hindawi Biomed Research Int 20 pages AID 2362108 (2018) https://doi.org/10.1155/2018/2362108

9. Z.Stefano, U.Veronesi, "Milestones in breast cancer treatment" the breast journal volume 21, issue 3, pages:3-12 (2015) doi:10.1111/tbj.12361

10. Mammographic Image Analysis Society, https://www.mammoimage.org/databases/

11. J.M.Masoud, E.Nasibeh, "A hybrid search method of wrapper feature selection by chaos particle swarm optimization and local search", Turk J Elec Eng \& Comp Sci volume: 24, pages: 3852-3861 (2016)

12. K.Ahmed, A.E.Hassanien, S.Bhattacharyya, "A Novel chaotic chicken swarm optimization algorithm for feature selection" pages: 259-264 IEEE (2017)

13. H.M.Zawbaa, E.Emary, C.Grosan, "Feature selection via Chaotic Ant lion optimization", Plosone volume (11) issue (3) (2016)

14. G.I.Sayed, A.E.Hassanien, A.T.Azar, "Feature selection via a novel chaotic crow search algorithm", Neural comp \&Appl , Springer (2017)

15. A.A.Ewees,M.A.E.Aziz, A.E.Hassanien, "Chaotic multi-verse optimizer based feature selection" Neural comp \& Appl, Springer (2017)

16. [16]Z.Srivastava, N.Sharma, S.K.Singh, R.Srivastava, "quantitative analysis of a general framework of a CAD tool for breast cancer detection from mammograms" J M.I pages 654-674 (2014)

17. [17]M.Pratiwi, J.Harefa, S.Nanda, "mammograms classification using grey level co-occurrence matrix and radial basis function neural network" Pro Cmp Sci pages 83-91 (2015)

18. S.Saini, R.Vijay, "Mammogram analysis using feed forward back propagation and cascade forward back propagation artificial neural network" Int Conf on Comm Sys \& N/w Tech IEEE pages 1177-1180 (2015) 
19. M.M Pawar, S.N.T, "Genetic fuzzy system based wavelet co-occurrence feature selection in mammogram classification for breast cancer diagnosis", P.Sci pages 247-250 (2016)

20. K.Vaidehi, T.S.S, "Automatic characterization of benign and malignant masses in mammography" Pro Com Science pages $1762-1769$ (2017)

21. A.Gautam, V.Bhateja, A.T, S.C.Satapathy, "An improved mammogram classification approach using back propagation neural network" D Eng \& Int Cmp pages 369-376 Springer (2018)

22. S.R.C.Sannasi, H.Rajaguru, "Comparison analysis of linear discriminants analysis and cuckoo search algorithm in the classification of breast cancer from digital mammograms" A.P.J.Cancer prevention volume 20 (2019)

23. N.J. Shelembijaphet, D.Xueweng, Y.Ayesha, "mammograms classification based on convolutional neural network" IOSR J Comp eng volume 22, issue 1, pages 44-49 (2020)

24. A.Krishnaveni, R.Shankar, S.Duraisamy, "An Efficient Methodology for Breast Tumor Segmentation using Duck Traveler Optimization Algorithm", PalArch's Journal of Archaelogy of Egypt/Egyptology, SCOPUS Indexed ISSN NO: 1567- 214X Volume 17, Issue 9, (2020).

25. A.Krishnaveni, R.Shankar, S.Duraisamy, "Duck Cluster Optimization Algorithm with K-Means Clustering for Mammogram Image Segmentation", Solid State Technology, SCOPUS Indexed ISSN NO: 0038-111X Volume 63, Issue 6, (2020).

26. Krishnaveni Arumugam, Shankar Ramasamy, Duraisamy Subramani "Improved Duck and Traveler Optimization (IDTO) Algorithm: A Two-way efficient approach for breast tumor segmentation using multilevel thresholding", European Journal of Molecular \& Clinical Medicine ISSN 2515-8260 Volume 7, Issue 10, 2020

27. Krishnaveni Arumugam, Shankar Ramasamy, Duraisamy Subramani "Binary Duck Traveler Optimization Algorithm for Feature selection in Breast Cancer Dataset Problem", IOT with Smart Systems - Proceedings of ICTIS 2021, Volume 2 (2021) SPRINGER (SCOPUS Indexed)

28. Krishnaveni A, Shankar R, Duraisamy S "Swarm Intelligence Algorithms with K-Means Clustering for Mammogram Image Segmentation”, IRJMETS, ISSN 2515-8260 Volume 3, Issue 2, (2021)

29. Krishnaveni A, Shankar R, Duraisamy S, " Enhanced Duck Traveler Optimization (EDTO) Algorithm with Multilevel Thresholding, Region of Interest and K-Means Clustering for Mammogram Image Segmentation to improving accuracy", International Conference on Research and development in science, Engineering and Technology (ICRDSET-2021).

30. Patel, G., DILIP B. Patel, and KINJAL M. Paghdal. "Analysis of P\&O MPPT algorithm for PV system." International Journal of Electrical and Electronics Engineering (IJEEE) 5.6 (2016) 1-10.

31. Khami, MOHAMMED JAWAR. "Unlimited Size of English Plain Text-in-Text Hiding Algorithm." International Journal of Computer Science and Engineering (IJCSE) 6.1 (2017) 89-96

32. Raja, M. Chithik, And M. Munir Ahmed Rabbani. "Performance Evaluation Of All Pair Shortest Path Parallel Algorithm Used In Big Data." International Journal Of General Engineering And Technology (Ijget) 6.4 (2017) 55-62

33. Onaizah, Ameer N. "A Novel Data Stream Clustering Algorithm In Healthcare Iot." Impact: International Journal Of Research In Applied, Natural And Social Sciences(Impact: Ijranss) 5.6 (2017) 51-60

34. Singh, Gurpreet, And Er Rajwinderkaur. "Review Paper On Decision Tree Data Mining Algorithms To Improve Accuracy In Identifying Classified Instances Using Large Dataset." International Journal Of Computer Science Engineering And Information Technology Research (Ijcseitr) 7.4 (2017)67-70

35. Azadeh, Yalda, and B. E. H. N. A. M. Mohammadi-Ivatloo. "Optimal Heat and Power Dispatch in Co-Generation Systems Using Firefly Algorithm." Tjprc: International Journal Of Power Systems \& Microelectronics (Tjprc: Ijpsm) 2.1 (2016) 77-86 